\title{
Unidirectional optical Bloch oscillations in asymmetric waveguide arrays
}

\author{
Pradeep Kumar and Miguel Levy* \\ Department of Physics, Michigan Technological University, Houghton, Michigan 49931, USA \\ *Corresponding author: mlevy@mtu.edu
}

Received August 2, 2011; revised October 4, 2011; accepted October 4, 2011;

posted October 7, 2011 (Doc. ID 152208); published November 11, 2011

\begin{abstract}
We present an analytical proof of the existence of unidirectional optical Bloch oscillations in a waveguide array system. It is shown that the presence of nonreciprocity in the system allows for a complete normal-mode dephasing in one of the propagation directions, resulting in a unidirectional breakdown in Bloch oscillations. A model system consisting of an array of transversely magnetized asymmetric $\mathrm{Si} / \mathrm{SiO}_{2}$ waveguides with a magneto-optic cover layer is presented. Large index contrasts between film and cover are critical for practical realizations. (C) 2011 Optical Society of America

OCIS codes: $\quad 130.2790,130.3120,230.7370,000.1600$.
\end{abstract}

In the 1930s Bloch and Zener predicted the phenomenon of Bloch oscillations (BOs), comprising oscillatory behavior of quantum particles in a periodic potential subject to constant external force [1]. The first experimental evidence confirming BOs came in 1960 when Chynoweth et al. observed an evenly spaced energy spectrum, the socalled Wannier-Stark ladder, for electrons in a crystalline medium placed in an external field [2]. Since then theoretical and experimental results showing similar nonclassical behavior in different particle systems, such as electrons in semiconductor superlattices, cold atoms in optical lattices, and electromagnetic waves in periodic dielectric systems, have been reported [3-6] . In the last two decades the idea of a discrete optical system (e.g., a waveguide array) exhibiting diffractionless propagation of an optical beam has drawn attention to the possibility of visualizing the oscillatory motion in the spatial domain with controlled beam dynamics [5-8].

In this Letter we extend the $\mathrm{BO}^{-}$phenomenon to unidirectional propagation in optical media. Prior work on optical BOs has dealt mostly with reciprocal phenomena [5-7]. In a previous Letter we examined the conditions for nonreciprocal BOs [8]. Recently, Longhi [7] predicted unidirectional BOs as a result of gain and/or loss in pseudo-Hermitian systems of complex optical lattices. Here we demonstrate theoretically that in the absence of gain or loss it is possible to attain cancellation of Bloch oscillatory motion in the optical regime unilaterally for one propagation direction while preserving this phenomenon in the opposite direction. We consider wave propagation in optical media characterized by gyrotropic dielectric permittivity tensors that, although possessing imaginary off-diagonal components, are strictly Hermitian in character. This study is realized for silicon platforms in a semiconductor-dielectric hybrid material system, where we examine a nontrivial case for a waveguide array structure exhibiting unidirectional optical BOs.

We thus extend our previous treatment [8] of different $\mathrm{BO}$ periods in opposite propagation directions to the case where complete cancellation of oscillatory motion occurs in one direction. Moreover, unidirectional BO is demonstrated here for a Si platform rather than the garnet platform in our prior work on nonreciprocal BOs. A key role is played by the TM mode nonreciprocal phase shift (NRPS) effect, which is due to the confinement of light in magnetized asymmetric waveguides lacking spatial inversion symmetry. This NRPS effect critically depends on the introduction of unequal spatial gradients in both the gyrotropy parameter and the refractive index across dissimilar waveguide interfaces [9].

The array structure used in the present study consists of ridge waveguides in silicon-on-insulator (SOI) with a bonded or sputter-deposited cerium-substituted yttrium iron garnet (Ce:YIG) cover [10]. An SOI-based garnet structure with a high index contrast and a large NRPS effect is best suited for the proposed unidirectional BO phenomenon. In the following we analytically establish the unidirectional character of optical BOs in the garnet/ SOI material system.

We consider an asymmetric one-dimensional waveguide array as shown in Fig. 1(a). This array serves as a periodic potential system to the optical beam, while a transverse index ramp acts as a lateral driving force on the photons. The index ramp is introduced through a constant difference in mode index between adjacent waveguides, which can be tuned by tailoring the width and/or height of the ridges $[\underline{6}, \underline{8}]$. Using the nearest-neighbor tight

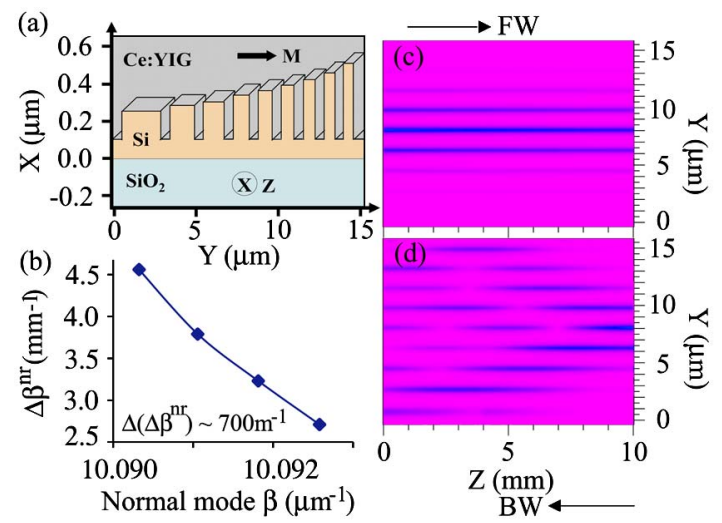

Fig. 1. (Color online) (a) Waveguide array (widths are scaled up and separations scaled down $4 \times$ to fit in the sketch), (b) plot showing nearly equally spaced NRPS $\Delta \beta^{n r}$ and propagation constant $\beta$ for adjacent normal modes; a typical forward normal mode of the array showing (c) constant amplitude and width in the FW direction and (d) diffractive spread in the BW direction. 
binding approximation and assuming $\mathrm{cw}$ propagation with no absorption, coupled-mode theory yields the following equation of motion for the optical field [5]:

$$
i \frac{d a_{n}^{f, b}}{d z}+\delta \beta^{f, b} n a_{n}^{f, b}+\kappa^{f, b}\left(a_{n-1}^{f, b}+a_{n+1}^{f, b}\right)=0,
$$

where $a_{n}^{f, b}$ describes the field amplitude in the $n$th waveguide, $\kappa^{f, b}$ is the interwaveguide coupling constant, and $f$ and $b$ stand for forward (FW) and backward (BW) direction of propagation. Upon excitation of a single central waveguide, the field evolution described by Eq. (1) results in localized beam oscillations with period $L_{B}=$ $2 \pi / \delta \beta$ and a lateral beam spread of about $\pm 4 \kappa / \delta \beta$ waveguides [5], where $\delta \beta=(2 \pi / \lambda) \cdot \Delta n_{\text {eff }}$ is the interwaveguide wavenumber difference, $\lambda$ is the wavelength in vacuum, and $\Delta n_{\text {eff }}$ is the modal refractive index difference.

We adopt a normal-mode approach to describe the beam dynamics across the array, with the $m$ th normalmode propagation constant given by $\beta_{0}+m \delta \beta$, where $\beta_{0}$ is the propagation constant of the zeroth mode. These normal modes are the Wannier-Stark states of the array structure. Their coherent movement depicts a bounded oscillatory path, with spatial Bloch period $L_{B}$. Here we show that it is possible to limit this oscillatory motion to only one direction of propagation utilizing the NRPS effect induced by a transversely magnetized cover layer.

For the beam propagation in the $z$-direction and a transverse in-plane magnetic field $\mathbf{M}$ in the $y$-direction [see Fig. 1(a)], the dielectric tensor of the magnetic cover layer is given by

$$
\hat{\varepsilon}=\left(\begin{array}{ccc}
\varepsilon & 0 & i g \\
0 & \varepsilon & 0 \\
-i g & 0 & \varepsilon
\end{array}\right),
$$

where $\varepsilon$ is the isotropic dielectric constant and $g$ is the gyrotropy parameter. Assuming no optical absorption (i.e., $\varepsilon$ and $g$ are real numbers), we treat the array system as optically lossless. For a planar waveguide made of an SOI substrate with a magnetic garnet cover layer, the TM mode dispersion relation is given by []]

$$
k_{x} \cdot d=\tan ^{-1}\left[\frac{\varepsilon_{f}}{k_{x}}\left(\frac{\gamma_{\mathrm{eff}}}{\varepsilon_{\mathrm{eff}}}+\frac{\beta g}{\varepsilon \varepsilon_{\mathrm{eff}}}\right)\right]+\tan ^{-1}\left[\frac{\varepsilon_{f}}{k_{x}}\left(\frac{\gamma_{s}}{\varepsilon_{s}}\right)\right],
$$

where $\gamma_{\text {eff }, s}=\left(\beta^{2}-k_{0}^{2} \varepsilon_{\text {eff }, s}\right)^{1 / 2}, k_{x}=\left(k_{0}^{2} \varepsilon_{f}-\beta^{2}\right)^{1 / 2}, \varepsilon_{\text {eff }}=$ $\varepsilon-g^{2} / \varepsilon, k_{0}$ is the vacuum wavenumber $(2 \pi / \lambda), \varepsilon_{s}$ is the dielectric constant of the substrate, $d$ is the thickness, and $\varepsilon_{f}$ is the dielectric constant of the core layer. Equation (2) gives a linear dependence in $\beta$, producing different solutions for FW and BW propagating waves, with propagation constants $\beta^{f}$ and $\beta^{b}$, where $\Delta \beta^{n r}=\beta^{f}-\beta^{b}$ is the NRPS per unit length. $\beta^{b}$ can be calculated by reversing the sign of $g$ (magnetization reversal). For all nine ridge waveguides in the array, an initial estimate of the propagation constant and the film thickness was made using Eq. (2). We note that this equation is only strictly valid for an infinite slab waveguide with the same substrate, film, and cover indices as the ridge waveguides considered here. However, finite ridge-width effects were taken into account through a perturbation theory approach [9] using the following:

$$
\Delta \beta^{n r}=\frac{2 \operatorname{Re} \iint \mathrm{d} x \mathrm{~d} y\left(\partial_{x} H_{y}\right) H_{y}^{*}\left(i g / \varepsilon^{2}\right)}{\iint \mathrm{d} x \mathrm{~d} y\left|H_{y}\right|^{2} \varepsilon^{-1}} .
$$

We use a three-dimensional (3D) semivectorial beampropagation method based on a finite difference algorithm to simulate the array model [8]. Mode indices and field profiles are obtained using the correlation method. For each normal mode, the calculated NRPS values were found to be varying approximately linearly with the propagation constants as shown in Fig. 1(b). The plot depicts nearly equally spaced $\beta$ and $\Delta \beta^{\text {nr }}$ values, representing the Wannier-Stark ladder in the waveguide array system [5].

Letting $\beta_{m}^{f}$ and $\beta_{m}^{b}$ be the propagation constants for FW and BW propagation for normal-mode $m$, the difference in intermodal propagation constant between the two directions is given by [8]

$$
\begin{aligned}
\delta \beta_{m+1, m}^{f}-\delta \beta_{m+1, m}^{b} & =\beta_{m+1}^{f}-\beta_{m}^{f}-\left(\beta_{m+1}^{b}-\beta_{m}^{b}\right) \\
& =\Delta \beta_{m+1}^{n r}-\Delta \beta_{m}^{n r}=\Delta\left(\Delta \beta^{n r}\right)_{m+1, m} .
\end{aligned}
$$

From Eq. (4) it is clear that in adjacent waveguides the difference between the propagation constants for FW and BW propagating waves is equal to the difference in nonreciprocal propagation constants. Equation (4) suggests that if one can design an array structure with intermodal wavenumber difference $\delta \beta^{f}=\Delta\left(\Delta \beta^{n r}\right)$, it is possible to have Bloch oscillatory motion in the FW direction with period $2 \pi / \Delta\left(\Delta \beta^{n r}\right)$, a characteristic feature of a discrete optical system [5]. In the BW direction the effect of $\delta \beta^{b}$ is completely cancelled out by the nonreciprocal $\Delta\left(\Delta \beta^{n r}\right)$, i.e., $\delta \beta^{b}=\delta \beta^{f}-\Delta\left(\Delta \beta^{n r}\right)=0$, resulting in a diffractive beam spread, analogous to the behavior of a homogenous array [5]. Precise tuning of the index gradient and NRPS allows unidirectional BOs. A large $\Delta\left(\Delta \beta^{n r}\right)$ is critical for practical realizations.

In the FW direction each forward normal mode maintains a constant amplitude and width $(\sim 10 \mu \mathrm{m})$, whereas in the BW direction it diffracts out [Figs. 1(c) and 1(d)]. The normal modes themselves do not exhibit BO motion. It is their superposition that does. Our designed array consists of nine waveguides [see Fig. 1(a)] made of a $\mathrm{Si}\left(n_{f}=3.44\right)$ core on a $\mathrm{SiO}_{2}\left(n_{s}=1.45\right)$ substrate with a Ce:YIG $\left(n_{c}=2.22\right)$ cover layer, wherein the thickness $(0.26$ to $0.5 \mu \mathrm{m})$ and the width $(0.6$ to $0.15 \mu \mathrm{m})$ have been adjusted to yield a constant $\delta \beta^{f}$ of about $700 \mathrm{~m}^{-1}$ in the FW direction. For a given design the wavelength tolerance is $4 \mathrm{~nm}$, and the tolerances in ridge height and width are $4 \%$ and $2 \%$, respectively. The interwaveguide separation is selected $(\sim 2 \mu \mathrm{m})$ to produce a constant coupling parameter $\kappa \sim 605 \mathrm{~m}^{-1}$. The ensuing $\delta \beta^{f}$ produces a spatial Bloch period $\left(L_{B}\right)$ of about $9 \mathrm{~mm}$ and a lateral beam spread of less than seven waveguides $(\sim 11 \mu \mathrm{m})$ for light propagating in the FW direction. Thus an array of nine coupled waveguides is enough to demonstrate the effect. A $0.2 \mu \mathrm{m}$ wide beam is launched into the center 


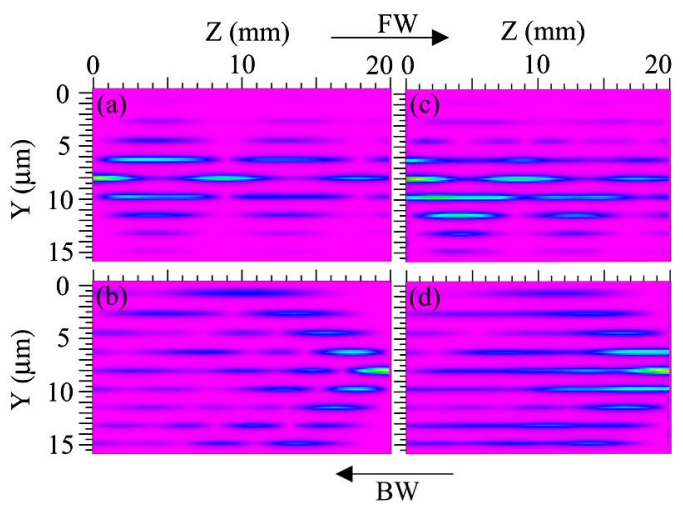

Fig. 2. (Color online) (a) Beam evolution for single guide excitation in the FW direction, showing BO motion, and (b) the BW direction, showing diffractive beam spread; (c) a $6 \mu \mathrm{m}$ wide beam excitation in the FW direction, showing BO motion with the beam mostly confined to the high index side; (d) diffractive beam spread in the BW direction.

waveguide, and the beam is allowed to propagate through the array. As expected, the beam exhibits BO motion with a period close to $9 \mathrm{~mm}$ and a lateral spread of $\sim 11 \mu \mathrm{m}$, as shown in Fig. 2(a). When a $6 \mu \mathrm{m}$ wide beam is launched at the input facet of the array, similar BO motion $\left(L_{B} \sim 9 \mathrm{~mm}\right)$ is observed, largely confined to the high wavenumber side with a lateral spread of $\sim 4$ waveguides [see Fig. 2(c)] [5] .

A large NRPS is introduced by adjusting the material index of each guide to create a $\Delta\left(\Delta \beta^{n r}\right)$ about $700 \mathrm{~m}^{-1}$. This is calculated by performing the integration in Eq. (3), for a typical value of $g \sim 0.0086$ at $\lambda=1.55 \mu \mathrm{m}$ for Ce:YIG. The large index contrast between the Si film and the Ce:YIG cover plays a critical role in realizing this strong NRPS. In the BW direction, the modification of interwaveguide $\delta \beta$ by the nonreciprocal $\Delta\left(\Delta \beta^{n r}\right)$ counters $\delta \beta^{f}$ and induces a $\delta \beta^{b} \sim 0$. Therefore, the index ramp vanishes, and an unlimited lateral beam spread is observed in the BW direction for both single guide excitation $(0.2 \mu \mathrm{m}$ wide) and broad beam excitation $(6 \mu \mathrm{m}$ wide), as expected $\left[L_{B}=2 \pi /\left(\delta \beta^{f}-\Delta\left(\Delta \beta^{n r}\right)\right) \sim \infty\right]$ and shown in Figs. 2(b) and 2(d), respectively.

We note here that the typical value of the NRPS effect for the garnet-based waveguides (e.g., $\mathrm{Bi}$ or rare-earthsubstituted YIG over $\mathrm{Gd}_{3} \mathrm{Ga}_{5} \mathrm{O}_{12}$ substrate) is very small $(\sim 1.4 \mathrm{rad} / \mathrm{mm})[9]$ and would require a very long array $(\sim 5 \mathrm{~cm})$ to demonstrate the proposed unidirectional effect. However, Si-based hybrid structures with magnetic garnet cover layers can offer an NRPS effect as large as $\sim 7.0 \mathrm{rad} / \mathrm{mm}$, as demonstrated in recent work by
Mizumoto and co-workers [10]. Our model system has a similar garnet/SOI composition, making possible the observation of the proposed unidirectional optical BO phenomenon within an array length of less than $1 \mathrm{~cm}$. Moreover, recent theoretical studies suggest NRPS values up to $100 \mathrm{rad} / \mathrm{mm}$ can be achieved by including a magneto-optical slot [11] or nanoscale air gap [12] in garnet/SOI waveguide designs. Such structures, yielding a high NRPS effect, would make a good choice for practical realization of the unidirectional $\mathrm{BO}$ phenomenon within micrometer-scale array lengths.

In summary, we demonstrate the existence of unidirectional BO in garnet/SOI waveguide media. It is shown that the counterpropagating waves behave completely differently, showing Bloch oscillatory motion in one direction and unlimited beam spreading in the opposite, by taking advantage of the TM-mode NRPS effect. Beyond fundamental studies, the unidirectional BO phenomenon offers potential applications in magnetooptically controlled one-way beam steering in all-optical integrated photonic devices. Furthermore, this effect could be employed for nonsaturated signal amplification in discrete array structures without disturbing the stability of the light source due to backreflection.

Work supported by National Science Foundation (NSF) Grant No. 0856650. The authors thank V. Belotelov and M. Vanwolleghem for a careful reading of the manuscript.

\section{References}

1. C. Zener, Proc. R. Soc. A 145, 523 (1934).

2. A. G. Chynoweth, G. H. Wannier, R. A. Logan, and D. E. Thomas, Phys. Rev. Lett. 5, 57 (1960).

3. C. Waschke, H. G. Roskos, R. Schwedler, K. Leo, H. Kurz, and K. Kohler, Phys. Rev. Lett. 70, 3319 (1993).

4. M. Ben Dahan, E. Peik, J. Reichel, Y. Castin, and C. Salomon, Phys. Rev. Lett. 76, 4508 (1996).

5. U. Peschel, T. Pertsch, and F. Lederer, Opt. Lett. 23, 1701 (1998).

6. R. Morandotti, U. Peschel, J. S. Aitchison, H. S. Eisenberg, and Y. Silberberg, Phys. Rev. Lett. 83, 4756 (1999).

7. S. Longhi, Phys. Rev. Lett. 103, 123601 (2009).

8. M. Levy and P. Kumar, Opt. Lett. 35, 3147 (2010).

9. R. L. Espinola, T. Izuhara, M.-C. Tsai, R. M. Osgood Jr., and H. Dötsch, Opt. Lett. 29, 941 (2004), and references therein.

10. H. Yokoi, Y. Shoji, and T. Mizumoto, Jpn. J. Appl. Phys. 43, 5871 (2004).

11. W. Zhang, J.-W. Mu, W.-P. Huang, and W. Zhao, Appl. Phys. Lett. 98, 171109 (2011).

12. R. Chen, G. Jiang, Y. Hao, J. Yang, M. Wang, and X. Jiang, Opt. Lett. 35, 1335 (2010). 